\title{
Check(point) or checkmate for acute myeloid leukemia?
}

\author{
Sandrine Niyongere ${ }^{1,2}$ and Aaron P. Rapoport ${ }^{1,2}$ \\ ${ }^{1}$ University of Maryland Marlene and Stewart Greenebaum Comprehensive Cancer Center and ${ }^{2}$ Department of Medicine, University of \\ Maryland School of Medicine, Baltimare, MD, USA \\ E-mail: AARON P. RAPOPORT - arapoport@umm.edu
}

doi:10.3324/haematol.2020.277103

I n this edition of Haematologica, Stroopinsky and colleagues report on the application of a personalized vaccine derived from fusing leukemia cells to autologous dendritic cells (fusion vaccine) as a potential tool to overcome checkpoint blockade in acute myeloid leukemia (AML). ${ }^{1}$ AML is an aggressive hematologic malignancy with poor long-term outcomes despite recent treatment advances. The only potential curative therapeutic option for intermediate- to high-risk AML is allogeneic stem cell transplantation through the induction of a graft-versus-tumor effect, demonstrating the importance of cell-based immunotherapy. ${ }^{2}$

Antibodies that block the programmed cell death protein 1 (PD-1) or programmed death ligand-1 (PD-L1) inhibitory pathway have led to improvements in progression-free survival and overall survival in several solid tumors and Hodgkin lymphoma, encouraging multiple clinical trials in other hematologic malignancies including AML. ${ }^{3}$ Daver and colleagues reported the overexpression of clinically targetable checkpoint inhibitor receptors, PD-1 and OX40, in the bone marrow of patients with AML, making checkpoint inhibition an interesting therapeutic option to study further. However to date, checkpoint inhibitor therapy for AML has yielded disappointing results. ${ }^{4,5}$

In an immunocompetent murine AML model using the TIB-49 murine AML cell line genetically altered to express luciferase and mCherry, Stroopinsky and colleagues confirmed that treatment with anti-PD1, anti-TIM3 or antirepulsive guidance molecule $b$ (RGMb) antibodies as single agents had little therapeutic efficacy compared to isotope controls. To overcome this resistance to checkpoint inhibition, these investigators tested a combinatorial approach whereby a fusion vaccine was given in combination with checkpoint inhibitors. Personalized vaccines derived from patient-derived AML cells fused with autologous dendritic cells have been previously tested in 17 patients who achieved complete remission after chemotherapy. ${ }^{6}$ Rosenblatt and colleagues observed that vaccination induced an increase in circulating T cells recognizing leukemia-specific antigens that persisted for more than 6 months with 12 of 17 patients remaining alive without recurrence at a median follow-up of 57 months. This study demonstrated that personalized vaccination of AML patients could induce expansion of leukemia-specific $T$ cells which may have the potential to protect against leukemia relapse.

In the study reported in this issue of the Journal, Stroopinsky and colleagues hypothesized that the combination of a personalized fusion vaccine and checkpoint inhibitor therapy could elicit a unique synergistic response whereby vaccination would induce leukemia-specific T-cell populations while checkpoint inhibition would enhance the function and persistence of these antileukemic $T$ cells. ${ }^{1}$ Using an immunocompetent murine AML model, cohorts of mice were vaccinated $24 \mathrm{~h}$ after being inoculated with murine leukemia cells followed by treatment with immune check- point inhibition every 3 days for a total of six doses. Rapid AML progression occurred by day 29 in all the control mice, which required euthanasia. Mice treated with checkpoint inhibition alone showed a modest improvement in survival compared to the control cohort, but all required euthanasia by day 44 . Two of five mice treated with the personalized vaccine alone remained leukemia free at day 90 of leukemia inoculation. Remarkably all the mice treated with vaccination and checkpoint blockade remained alive and leukemia free at day 90 after leukemia inoculation. Stroopinsky and colleagues showed that mice treated with the personalized vaccine alone had variable expansion of tumor reactive $T$ cells, but mice treated with the combination of personalized fusion vaccine and checkpoint blockade demonstrated robust expansion of circulating tumor-specific $\mathrm{CD}^{+} \mathrm{T}$ cells. The enhanced expansion of tumor-specific $T$ cells following vaccination and checkpoint blockade was confirmed in the splenocytes of mice euthanized 17 days after leukemia inoculation, which showed that the combination of vaccine with checkpoint inhibition resulted in induction of tumor-specific immunity with prevention of leukemia engraftment. The investigators further found that combination treatment with the fusion vaccine and checkpoint inhibition induced a T-cell memory response and increased clonal diversity along with a statistically significant decrease in $\mathrm{CD}^{+} / \mathrm{CD}^{2} 5^{+} \mathrm{FOXP}^{+} \mathrm{T}$ regulatory cells compared to treatment with either the fusion vaccine or checkpoint inhibition alone. Stroopinsky and colleagues also demonstrated that the combination approach provided long-term protection from leukemia relapse even after re-challenge via retro-orbital inoculation of a lethal dose of leukemia at day 90 after treatment.

Important questions remain, such as whether these impressive pre-clinical results can be replicated in a broader range of murine leukemias. It is also unclear whether this combination approach, which utilizes a fusion vaccine created with a "snapshot" of the leukemia at diagnosis, can elicit effective and long-term immune responses against the genetically complex and clonally heterogeneous leukemic populations that characterize AML in humans. Relapses of AML are often due to the emergence of treatment-resistant clones that may be undetectable at diagnosis and may not be sufficiently immunogenic in this model. Nonetheless the study reported in this issue of the Journal provides a strong scientific foundation for a clinical trial of combination therapy using personalized fusion vaccines and checkpoint inhibition. Results are eagerly awaited to determine whether this novel approach can finally check(point) or even checkmate AML.

\section{Disclosures}

No conflicts of interest to disclose.

\section{Contributions}

$S N$ and APR co-wrote the manuscript. 


\section{References}

1. Stroopinsky D, Liegal J, Bhasin M, et al. Leukemia vaccine overcomes limitations of checkpoint blockade by evoking clonal T-cell responses in a murine acute myeloid leukemia model. Haematologica;2021;106(5):1330-1342.

2. Alyea EP, Kim HT, Ho V, Corey Cutler, et al. Comparative outcome of nonmyeloablative and myeloablative allogeneic hematopoietic cell transplantation for patients older than 50 years of age. Blood. 2005;105(4):1810-1814.

3. Luksza M, Riaz N, Makarov V, et al. A neoantigen fitness model predicts tumor response to checkpoint blockade immunotherapy.
Nature. 2017:551(7681):517-520

4. Daver N, Basu S, Garcia-Manero G, et al. Defining the immune checkpoint landscape in patients (pts) with acute myeloid leukemia (AML). Blood. 2016; 128(22):2900.

5. Daver N, Garcia-Manero G, Basu S, et al. Efficacy, safety, and biomarkers of response to azacitidine and nivolumab in relapse/refractory acute myeloid leukemia: a nonrandomized, open-label, phase II study. Cancer Discov. 2019;9(3):370-383.

6. Rosenblatt J, Stone RM, Uhl L, et al. Individualized vaccination of AML patients in remission is associated with induction of antileukemia immunity and prolonged remissions. Sci Transl Med. 2016;8(368):368ra171.

\section{Immune thrombocytopenia in myeloid and lymphoid clonal disorders: an intriguing association}

\section{Francesco Rodeghiero}

Hematology Project Foundation - affiliated to the Hematology Department of San Bortolo Hospital, Vicenza, Italy E-mail: FRANCESCO RODEGHIERO - rodeghiero@hemato.ven.it

doi:10.3324/haematol.2020.275933

I $\mathrm{n}$ this issue of Haematologica, Jachiet et al. ${ }^{1}$ present the first systematic study on the association of severe immune thrombocytopenia (ITP) with preleukemic clonal myeloid disorders. Patients from 16 French Departments of Hematology and Internal Medicine were accrued between January 1999 and July 2019, under the coordination of the French Network of Dysimmune Disorders Associated with Hemopathies. A total of 41 cases, 17 with myelodysplastic syndrome (MDS) and 24 with chronic monomyelocytic leukemia (CMML), meeting the 2016 World Health Organization classification ${ }^{2}$ and a maximum period of 10 years between the diagnosis of ITP and MDS/CMML were retained for the final retrospective analysis. The majority of cases (73\%) were scored as low-risk with a median Revised International Prognostic Scoring System score of $3 .^{3}$ ITP, mainly of chronic type, was diagnosed with bona fide criteria and could be anterior, concomitant or posterior to the diagnosis of the myeloid disorder. These patients were compared to $200 \mathrm{MDS} / \mathrm{CMML}$ patients without ITP and to a control group of 75 patients with primary ITP without MDS/CMML

Patients with MDS/CMML with associated ITP had more severe bleeding and a multirefractory profile to firstline treatments for ITP compared to those with primary ITP alone and showed a moderate response to thrombopoietin-receptor agonists. They had a lower rate of progression toward acute myeloid leukemia than MDS/CMML patients without ITP but, disappointingly, the overall survival was similar. Limited cytogenetic and molecular studies did not contribute to differentiate MDS/CMML with or without ITP, apart from a higher prevalence of $20 q$ deletion in cases with ITP, but highthroughput next-generation sequencing was not used to describe genetic profiles.

In addition to these interesting clinical findings, the study by Jachiet et al. poses a preliminary question: is the "association" of ITP with low-grade myelodysplastic dis- orders (whichever comes first) just casual or is it indeed related to a shared pathogenic mechanism? In other words, is the prevalence of this association beyond what could be expected by chance alone?

Unfortunately, Jachiet et al. ${ }^{1}$ did not report the number of patients with MDS/CMML from which the ITP cases were identified, thus hampering any estimation of the prevalence of ITP associated with MDS/CMML, unlike another French study reporting 61 low-risk MDS patients in nine of whom (15\%) ITP was identified as the cause of thrombocytopenia (platelet count $<70 \times 10^{9} / \mathrm{L}$ ) on the basis of a greater reduction in platelet lifespan and low bone marrow blast infiltration $(<10 \%)$ not justifying the severity of the thrombocytopenia. Indeed, splenectomy was successful in three of these cases. ${ }^{4}$ A much lower percentage $(3 \%)$ of thrombocytopenia of putative autoimmune nature was identified among 1,408 MDS patients included in the Moffitt Cancer Center database and at King's College Hospital. ${ }^{5}$

Conversely, limited investigations have tackled the problem from the other side, by reporting the incidence of co-occurrence or subsequent development of MDS in patients first presenting with ITP. The only large study on this issue is based on the identification of 2,885 adults with incident ITP requiring healthcare and accessing the French health insurance national database over a 3-year period. ${ }^{6}$ Among these patients, $2.3 \%$ were concomitantly affected by MDS. Interestingly, some reports of "primary" ITP later developing into MDS are also available and it is noteworthy that in the study by Jachiet et al. ${ }^{1}$ ITP preceded the diagnosis of MDS/CMML in $36 \%$ of cases by several months to years. In another retrospective French series of 516 patients with ITP, the diagnosis of CMML was unveiled by the finding of thrombocytopenia in eight cases $(1.4 \%)$ and 13 additional cases were identified through a systematic literature review of patients in whom the diagnosis of CMML was associated with or heralded by (in some cases several years before) isolated 